\title{
GESTÃO DE ALTA PARA A CONTINUIDADE DO CUIDADO: EXPERIÊNCIA DAS ENFERMEIRAS DE LIGAÇÃO DE PORTUGAL
}

\author{
Maria Manuela Martins¹, Gisele Knop Aued², Olga Maria Pimenta Lopes Ribeiro ${ }^{3}$ \\ Maria José Santos ${ }^{4}$, Maria Ribeiro Lacerda ${ }^{5}$, Elizabeth Bernardino ${ }^{6}$
}

\begin{abstract}
Objetivo: descrever as atividades de continuidade do cuidado desenvolvidas pelas enfermeiras de ligação nos serviços hospitalar. Método: estudo descritivo, exploratório, realizado de setembro/2016 a março/2017, em 15 hospitais de Portugal, com 107 enfermeiras. Os dados foram coletados com um questionário semiestruturado e analisados por meio das frequências absolutas. Resultados:51 (47,7\%) dos pacientes que necessitam de cuidados continuados são identificados por médicos e enfermeiras assistenciais, 69 (64,5\%) utilizam critérios para a identificação dos riscos para a alta do paciente. Os elementos considerados no planejamento de alta são: reabilitação motora e funcional, 54 (30,86\%); e preparo do cuidador, $41(23,43 \%)$. As transferências das informações para continuidade do cuidado ocorrem majoritariamente por correio eletrônico, 58 (25,55\%). Conclusão: as atividades para a continuidade do cuidado não são homogêneas entre os profissionais que realizam atividades de ligação, mas contribuem para estratégias mais consolidadas para o enfrentamento da descontinuidade do cuidado.

DESCRITORES: Enfermagem; Gerenciamento da prática profissional; Continuidade da assistência ao paciente; Alta do paciente.
\end{abstract}

\section{DISCHARGE MANAGEMENT TO ENSURE CONTINUITY OF CARE: EXPERIENCE OF PORTUGUESE LIAISON NURSES}

Objective: To describe the activities performed to ensure continuity of care by liaison nurses in hospital services. Method: Descriptive exploratory study with 107 nurses conducted from September 2016 to March 2017, in 15 hospitals in Portugal. Data was collected with a semi-structured questionnaire and the absolute frequencies were calculated for data analysis. Results: Fifty-one $51(47.7 \%)$ patients who require continuous care were identified by physicians and nursing assistants; $69(64.5 \%)$ health professionals used specific criteria to identify the discharge risk factors. The elements considered in the discharge planning are motor and functional recovery; 54 (30.86\%); and caregiver's capacity to provide caregiving activities, 41 (23.43\%). Most information related to care continuity is exchanged by email, 58 (25.55\%). Conclusion: The activities associated with continuity of care are not homogeneous among the professionals who carry out liaison activities, but contribute to the use of more consistent strategies for coping with discontinuity of care.

KEYWORDS: Nursing; Management of professional practice; Continuity of patient care; Patient discharge.

\section{GESTIÓN DEL ALTA PARA CONTINUIDAD DE LA ATENCIÓN: EXPERIENCIA DE ENFERMERAS DE COMUNICACIÓN EN PORTUGAL}

Objetivo: describir las actividades de continuidad de la atención desarrolladas por las enfermeras de comunicación en el servicio hospitalario. Método: estudio descriptivo, exploratorio, realizado de setiembre/2016 a marzo/2017 en 15 hospitales de Portugal con 107 enfermeras. Datos recolectados mediante cuestionario semiestructurado, analizados por frecuencias absolutas. Resultados: 51 (47,7\%) de los pacientes que precisaban atención continuada son identificados por médicos y enfermeras de atención; $69(64,5 \%)$ utilizan criterios de identificación de riesgos para el alta del paciente. Los elementos considerados en la planificación del alta son: rehabilitación motriz y funcional, 54 (30,86\%); y capacitación del cuidador $41(23,43 \%)$. La transferencia de información para continuidad del cuidado sucede mayormente vía correo electrónico, 58 (25,55\%). Conclusión: las actividades para continuidad de la atención no son homogéneas entre los profesionales actuantes en la comunicación, pero colaboran con estrategias más sólidas para enfrentar la discontinuación del cuidado.

DESCRIPTORES: Enfermería; Gestión de la Práctica Profesional; Continuidad de la Atención al Paciente; Alta del Paciente.

${ }^{1}$ Enfermeira. Doutora em Enfermagem. Docente da Escola Superior de Enfermagem do Porto. Porto- Portugal.

${ }^{2}$ Enfermeira. Doutora em Enfermagem. Universidade Federal do Paraná. Curitiba, PR, Brasil.

${ }^{3}$ Enfermeira. Doutoranda em Ciências da Enfermagem. Universidade do Porto. Instituto de Ciências Biomédicas Abel Salazar. Porto, Portugal.

${ }^{4}$ Enfermeira. Mestre em Direção e Chefia dos Serviços de Enfermagem. Escola Superior de Enfermagem do Porto. Porto, Portugal.

${ }^{5}$ Enfermeira. Doutora em Enfermagem. Docente do Programa de Pós- Graduação em Enfermagem da Universidade Federal do Paraná. Curitiba, PR, Brasil.

${ }^{6}$ Enfermeira. Doutora em Enfermagem. Docente do Programa de Pós- Graduação em Enfermagem da Universidade Federal do Paraná. Curitiba, PR, Brasil. 


\section{INTRODUÇÃO}

A continuidade do cuidado é definida pelo Serviço Nacional de Saúde (SNS) português como a sequencialidade, no tempo e nos sistemas de saúde e segurança social, das ações integradas de saúde e apoio social. (1) É uma estratégia e uma política a ser seguida pelos serviços de saúde, pois se associa à melhoria da qualidade dos cuidados em saúde, ${ }^{(2)}$ minimiza custos ${ }^{(3)}$ reduz hospitalizações evitáveis entre idosos ${ }^{(4)}$ e diminui riscos de hospitalização. ${ }^{(5)}$

Os pacientes vivenciam descontinuidade do cuidado quando há mudanças entre os serviços e quando transitam entre as instituições de saúde.(2) Assim, para assegurar a continuidade do cuidado, o SNS português conta com a Rede Nacional de Cuidados Continuados Integrados (RNCCI) ${ }^{(6)}$ voltada às pessoas em situação de dependência que necessitam de cuidados continuados de saúde e de apoio social, de origem preventiva, reabilitadora ou paliativa. As unidades que compõem a RNCCI compreendem as unidades de internamento, ambulatoriais, equipes hospitalares e domiciliares. ${ }^{(7)}$

O papel da RNCCI é dar resposta na reabilitação dos clientes dependentes depois de um episódio agudo, capacitar os familiares que não adquiriram competências para cuidar durante o internamento e/ ou aguardar a preparação do doente para ser internado numa residência de idosos.

Os sistemas de referenciação para a continuidade do cuidado, em Portugal, são as famílias, as unidades da RNCCI e a comunidade, principalmente as Unidades de Saúde da Família (USF). Desta forma, a fim de uniformizar o processo de referenciação dos pacientes do hospital para a RNCCI, ou para os demais sistemas de referenciação, cada hospital do SNS português possui uma Equipe de Gestão de Altas (EGA). A EGA é multidisciplinar, formada no mínino por um enfermeiro, um médico e um assistente social, com o objetivo de avaliar e confirmar a proposta de referenciação dos pacientes demandadas pelas equipes assitenciais. ${ }^{(8)}$

Para proporcionar a continuidade do cuidado no modelo de gestão de altas português, além das enfermeiras que ocupam um cargo na EGA, há enfermeiras assistenciais e/ou gestores que tem a função de ligação. ${ }^{(9)}$ As enfermeiras assistenciais identificam e avaliam os pacientes que necessitam de continuidade de cuidado e, então, são elos entre os serviços hospitalares e a EGA, ou entre os serviços hospitalares e a comunidade/família, nas situações em que os pacientes não são referenciados para unidades da RNCCl. Já as enfermeiras da EGA realizam o plano e a transferência do paciente dos serviços hospitalares para os sistemas de referenciação externos, principalmente para a RNCCI.

A EGA de cada hospital trabalha em parceria com a equipe assistencial das diferentes unidades hospitalares, de forma a planejar a alta dos pacientes, considerando suas necessidades. É fundamental que as enfermeiras da equipe de gestão de altas e as enfermeiras assistenciais trabalhem em equipe e tenham objetivos claros acerca da necessidade da continuidade do cuidado, bem como os meios e estratégias para alcançá-la.

Sustenta-se que o conhecimento das atividades desenvolvidas pelas enfermeiras que desempenham a função de ligação no SNS português pode ser útil para delinear estratégias de enfrentamento da descontinuidade do cuidado no contexto brasileiro, evidenciada pela fragilidade da articulação entre os serviços de diferentes níveis assistenciais e a deficiência no sistema de contrarreferência. ${ }^{(10-11)}$

Este estudo faz parte de um projeto multicêntrico, pioneiro na realidade brasileira, que buscou experiências das enfermeiras de ligação em Portugal, no Canadá e na Espanha, com o intuito de elucidar e subsidiar a atuação da enfermeira de ligação no Brasil, como profissional com capacidade de contribuir efetivamente para a continuidade do cuidado. ${ }^{(12)}$

Assim, tem-se como objetivo descrever as atividades de continuidade do cuidado desenvolvidas pelas enfermeiras de ligação nos serviços hospitalares. 


\section{MÉTODO}

Trata-se de um estudo descritivo, exploratório, de abordagem quantitativa, desenvolvido em 15 hospitais públicos da região norte de Portugal continental, do qual participaram enfermeiras da EGA e enfermeiras assistenciais e/ou gestores que desempenhavam a função de ligação, totalizando 107 enfermeiras.

Foram critérios de inclusão: ser enfermeira e elo para a gestão da alta hospitalar dos serviços de Medicina e Cirurgia das instituições citadas.

As enfermeiras foram convidadas a participar da pesquisa por uma pesquisadora portuguesa que garantiu o anonimato das participantes, explicou os objetivos, entregou o Termo de Consentimento Livre e Esclarecido e o questionário semiestruturado de autopreenchimento. A coleta de dados aconteceu no próprio estabelecimento de trabalho, com duração de 15 a 20 minutos, de setembro de 2016 a março de 2017.

O instrumento de coleta de dados foi o mesmo utilizado nos três países citados no estudo multicêntrico. Fizeram-se necessárias pequenas adaptações transculturais,a fim de ajustar termos para o português de Portugal e evitar vieses. O questionário semiestruturado passou pela validação de conteúdo por cinco enfermeiras portuguesas, uma pesquisadora e quatro gestoras de serviços de hospitais, que não participaram do estudo. As questões buscaram explicitar o perfil das participantes da pesquisa, a identificação do paciente que necessita da continuidade do cuidado, a avaliação inicial do paciente, o planejamento da alta hospitalar, bem como a transferência das informações entre o hospital e os serviços extra-hospitalares.

No Brasil, o projeto de pesquisa foi aprovado pelo Comitê de Ética em Pesquisa da Universidade Federal do Paraná sob o número 1.426.575. Em Portugal, passou pelos pareceres da Comissão de Ética para a Saúde do Centro Hospitalar de São João e da Faculdade de Medicina da Universidade do Porto, tendo sido aprovado com o parecer n.ำ 157/16. Posteriormente, foi enviada solicitação de autorização para a pesquisa a cada instituição participante conjuntamente com os pareceres do Brasil e de Portugal. Dentre os 18 hospitais convidados, três não aceitaram participar.

Após a coleta de dados, os mesmos foram digitados em uma planilha no programa Excel 2010,analisados e discutidos a partir das frequências absolutas obtidas.

\section{RESUlTADOS}

\section{Perfil dos participantes}

Dentre os 107 participantes da pesquisa, 83 (77,6\%) eram do sexo feminino, com idade média de 48 anos. Quanto ao tempo de exercício profissional, a variação foi de 1 a 40 anos, sendo que a média foi de 19,7 anos. Em relação à área de atuação, do total de participantes, 98 (91,6\%) eram assistenciais e/ou gestores e desempenhavam a função de ligação, 9 (8,4\%) eram coordenadores da EGA. Em relação ao grau de instrução, 79 (73,8\%) possuíam licenciatura em Enfermagem, 26 (24,3\%) mestrado e 2 (1,9\%) eram doutoras.

\section{Identificação do paciente que necessita de cuidados continuados após a alta hospitalar}

A necessidade de cuidados continuados após a alta hospitalar, para os participantes, é identificada por médicos e enfermeiras, $51(47,7 \%)$; seguida pelos demais profissionais da equipe assistencial, 42 $(39,3 \%)$; e $8(7,5 \%)$, exclusivamente pelos médicos.

Do total de participantes, 69 (64,5\%)utilizam critérios para facilitar a identificação de potenciais riscos ou problemas para a alta do paciente, 27 (25,5\%) não utilizam nenhum critério e os demais referem que a utilização de critérios não se aplica. 
Quando utilizados, os critérios para facilitar a identificação de potenciais riscos ou problemas para a alta do paciente são: viver sozinho, 103 (97,2\%); dependência para o autocuidado, 101 (94,4\%); ter múltiplas lesões por pressão, 90 (91,8\%); ser indigente, 95 (91,3\%); ser vítima de maus tratos ou negligência, 94 (90,4\%); ter registro de ocorrência de quedas no domicílio, 82 (85,4\%); e ter doenças crônicas agudizadas, 83 (88,3\%).

O meio mais utilizado entre os profissionais da EGA, enfermeiros assistenciais e gestores, para trocar informação necessária para a continuidade do cuidado internamente são: formulário específico impresso, 50 (46,7\%); associação do telefone, correio eletrônico e formulário impresso, 31 (29\%); e associação do telefone e formulário específico impresso, 25 (23,4\%).

\section{Avaliação inicial do paciente}

O processo de avaliação inicial do paciente, em busca de critérios que atendam às necessidades futuras de cuidados continuados, pode ser realizado tanto pelas enfermeiras da EGA quanto pelas enfermeiras assistências e consiste em exame físico, coleta de dados no prontuário e entrevista. Esta última é feita por $75(70,1 \%)$ das enfermeiras, $13(12,1 \%)$ não a realizam e $19(17,8 \%)$ responderam que não se aplica.

Para aqueles que realizam as entrevistas, são abordados o motivo da internação, antecedentes, contexto e histórico familiar, hábitos de vida, estado atual, uso de medicação, grau de dependência motora e funcional, risco de quedas e de úlceras de pressão, capacidade de decisão do paciente, avaliação da deglutição, do equilíbrio e do componente sensorial, necessidades após a alta hospitalar, capacitação do cuidador principal, conhecimento do paciente e do cuidador acerca da prevenção de complicações e condições habitacionais.

O exame físico é realizado por 78 (72,9\%) participantes. Aqueles que não realizam exame físico,13 $(12,1 \%)$, não o fazem porque atribuem esta tarefa ao enfermeiro responsável pelo paciente, ou responsável pela admissão (conforme norma nacional), ou, ainda, porque são gestores ou membros da EGA e utilizam as informações dos exames físicos realizados pelos enfermeiros assistenciais registradas em prontuário.

Em relação à participação de um familiar na avaliação inicial do paciente,74 (69,2\%) das enfermeiras habitualmente realizam o contato com um familiar, $13(12,1 \%)$ não realizam tal contato e as demais referiram que não se aplica.

\section{Planejamento da alta hospitalar}

O planejamento da alta, que consiste em avaliação, preenchimento de formulário e previsão da data de alta, é realizado nas primeiras 48 horas após o internamento por $71(66,4 \%)$ dos participantes. Para $30(28 \%)$, a antecipação não ocorre e, portanto, a necessidade de continuidade de cuidado é verificada no momento da alta hospitalar;3 $(2,8 \%)$ responderam que o planejamento da alta é iniciado em outro momento, não especificando em qual momento; e 3(2,8\%) não responderam.

Para $102(95,3 \%)$ participantes, o planejamento de alta ocorre com envolvimento de outros profissionais, por meio de reuniões para discussão de casos;2 $(1,9 \%)$ responderam que não há o envolvimento de outros profissionais para além das enfermeiras assistenciais;e os demais participantes não responderam.

Os profissionais que podem ser envolvidos no planejamento da alta para os participantes são: enfermeiras assistenciais, 84 (78,5\%); médicos, 85 (79,4\%); assistentes sociais, 96 (89,7\%); enfermeiras da EGA, 85(79,4\%);nutricionistas, 30 (27,1\%); fisioterapeutas,8 (7,5\%); psicólogo,3 (2,8\%); e fisiatras,2 (1,9\%).

Em relação à existência de um coordenador do planejamento da alta, 49 (45,8\%) responderam que há um coordenador, $44(41,1 \%)$ referiram que não há, $13(12,1 \%)$ apontaram que não se aplica e 1 participante não respondeu. Quanto ao profissional que coordena o planejamento da alta, para $15(14 \%)$ participantes esta é uma atribuição da enfermeira da EGA, para $12(11,2 \%)$ o médico, para 10 (9,3\%) é a enfermeira assistenciale para $1(0,9 \%)$ outro profissional não especificado pelo participante da pesquisa. 
Os principais elementos incluídos no planejamento da alta resultante da avaliação inicial são apresentados na Tabela 1.

Tabela 1- Principais elementos do planejamento da alta hospitalar. Porto, Portugal, 2016-2017

\begin{tabular}{lcc}
\hline Principais elementos do planejamento de alta & $\mathbf{n}$ & $\mathbf{\%}$ \\
\hline Preparo do cuidador & 41 & 23,43 \\
Reabilitação motora e funcional & 54 & 30,86 \\
Gestão do regime medicamentoso & 34 & 19,43 \\
Tratamento de lesões por pressão & 02 & 1,14 \\
Recuperação da autonomia prévia & 01 & 0,57 \\
Treino do autocuidado & 33 & 18,86 \\
Grau de dependência & 02 & 1,14 \\
Cuidados a seguir & 06 & 3,43 \\
Principais diagnósticos de enfermagem & 01 & 0,57 \\
Situação social & 01 & 0,57 \\
\hline Total & 175 & 100 \\
\hline
\end{tabular}

Para $98(91,6 \%)$ dos participantes,um documento, com informações sobre as estratégias para a continuidade do cuidado,é entregue ao paciente no momento da alta; para 7 (6,5\%), não se aplica; e os demais não responderam.

Em relação ao contato com o familiar do paciente no dia da alta hospitalar, 96 (89,7\%) dos participantes afirmam haver alguma comunicação. Esta comunicação é realizada para informar sobre o momento da alta, repassar as informações referentes aos cuidados desenvolvidos durante o internamento, bem como capacitar os familiares quanto aos cuidados para com o paciente após a alta, $3(2,8 \%)$ não realizam comunicação; para 7 (6,5\%), não se aplica; e1 participante não respondeu.

\section{Transferência das informações entre o hospital e os demais serviços extra-hospitalares}

Os meios da transferência de informações entre o hospital e os serviços extra-hospitalares são mostrados na Tabela 2.

Tabela 2- Principais formas de transferência das informações. Porto, Portugal, 2016-2017

\begin{tabular}{lcc}
\hline Formas de transferências das informações & $\mathbf{n}$ & $\mathbf{\%}$ \\
\hline Troca de e-mail entre os profissionais & 58 & 25,55 \\
Contato telefônico entre os profissionais & 55 & 24,23 \\
Carta entregue pelo paciente & 44 & 19,38 \\
Plano de alta entregue pelo paciente & 27 & 11,89 \\
Formulário impresso & 1 & 0,44 \\
Sistema informático & 41 & 18,06 \\
Outra forma & 1 & 0,44 \\
\hline Total & 227 & 100 \\
\hline
\end{tabular}


Os sistemas de referência para a continuidade de cuidados, normalmente são: a família, as unidades que compõem a RNCCl e as USF. De acordo com os participantes, os profissionais das USF e da RNCCI recebem as informações do paciente no dia em que o paciente recebeu a alta hospitalar, 40 (37,4\%);um dia antes da alta hospitalar, 34 (31,8\%); um dia depois da alta, 10 (9,3\%); ou, menos frequentemente, uma semana antes da alta, $3(2,8 \%)$. Ainda há casos em que os profissionais que recebem os pacientes referenciados não são informados, 14 (13,1\%).

\section{DISCUSSÃO}

A identificação dos pacientes que necessitam de cuidados continuados após a alta hospitalar deve ser realizada, preferencialmente, durante as primeiras 48 horas de internamento do paciente. ${ }^{(7)} \mathrm{O}$ que parece estar sendo realizado, pois mais da metade dos participantes da pesquisa $(66,4 \%)$ indicaram que o planejamento de altas e inicia nas primeiras 48 horas da internação do paciente. Um dos elementos que garante a continuidade dos cuidados entre os diferentes níveis assistenciais é iniciar o planejamento da alta desde o início da internação do paciente. ${ }^{(13)}$

A identificação do paciente que necessita de cuidados continuados pode ser realizada por diferentes profissionais. Assim, a ligação com a EGA poder ser estabelecida pelas enfermeiras assistenciais e/ ou pelas enfermeiras gestores, uma vez que nem todos os serviços contam com um coordenador do planejamento da alta. A ausencia deste coordenador pode contribuir para a subnotificação dos pacientes à EGA, dispersar informações e impossibilitar o planejamento da alta em tempo oportuno.

Dentre os critérios utilizados para a identificação dos pacientes que necessitam de cuidados continuados e são referenciados para a RNCCl ou demais sistemas de referenciação, viver sozinho, ocorrência de quedas e dependência para o autocuidado vão ao encontro de um estudo que abarca o perfil dos pacientes mais admitidos na $\mathrm{RNCCl}$ : idosos, dependentes ou incapazes quanto à autonomia física, pessoas do sexo feminino,sem qualificação profissional e com até seis anos de escolaridade. (14) Verifica-se, ainda, que na avaliação inicial do paciente a maioria dos dados coletados é útil para a continuidade dos cuidados, já que inclui informações tanto clínicas quanto sociais.

Orecurso mais utilizado pelas enfermeiras assitenciais para socilitar que os cuidados sejam continuados, ou seja, informar à EGA,é o formulário específico impresso, mesmo com sistema informático implantado em todos os hospitais que participaram do estudo. O trabalho em equipe na saúde é complexo, sendo a comunicação efetiva um ponto-chave que demanda avaliação contínua, em que incida normas, valores, hábitos, regras e experiências vivenciadas e partilhadas pelos gestores e profissionais da equipe. ${ }^{(15)}$ Ter um canal definido para que esta comunicação aconteça é fundamental para a continuidade do cuidado.

A enfermeira da EGA foi o profissional que se destacou como coordenador do planejamento da alta hospitalar, o que pode associar-se ao fato da EGA ser responsável pela referenciação dos pacientes à RNCCI. ${ }^{(8)}$ Além disso, os enfermeiros são reconhecidos pela capacidade de compreender a pessoa como um todo, pela integralidade da assistência à saúde, pela habilidade de acolher e identificar-se com as necessidades e expectativas dos pacientes e seus familiares, bem como pela capacidade de otimizar as intervenções de cuidado em saúde de forma que integre e abarque os saberes profissionais, dos usuários e da comunidade. ${ }^{(16)}$

Dentre os principais elementos incluídos no planejamento da alta hospitalar, salienta-se a reabilitação motora e funcional, fundamental para minimizar as limitações relacionadas às atividades de vida diária (AVD) dos pacientes. Neste sentido, estudo que avaliou a capacidade funcional nas áreas de recursos sociais, econômicos, saúde mental, saúde física e AVD de pessoas com 75 anos ou mais do conselho de Coimbra demonstrou que a maioria, 45,6\%, apresentou limitação grave ou total na área da saúde física, seguida de 23,6\% com limitação grave ou total quanto às AVD. ${ }^{(17)}$

O preparo do cuidador é essencial para os cuidados contínuos e é realizado ao identificar a possibilidade de reabilitação do paciente, alto grau de dependência, debilidade econômica, entre outros. É preciso considerar a transição de um estado de saúde para um de estado de doença e dependência, de pessoas ou equipamentos, que o paciente vivencia, mas também avaliar a transição situacional dos familiares para o papel de cuidadores no domicílio e a importância de capacitá-los para o exercício desse papel ${ }^{(18)}$ da melhor forma possível. 
O regime medicamentoso de uso pós-alta sofre alterações durante a internação com inclusões ou exclusões, que podem mudar inclusive a rotina dos pacientes. Objetivando a continuidade do cuidado na comunidade ou domicílio,os profissionais da atenção primária solicitam acuradas informações acerca da mudança de medicamentos dos pacientes que passaram por uma internação e, ainda, pedem lista completa dos medicamentos prescritos na alta hospitalar. ${ }^{(13)}$ Intervenções para melhorar a adesão terapêutica é um importante subsídio para a melhoria da saúde da população idosa, com reflexos no aumento da qualidade de vida, redução das recorrências aos serviços de urgência e de hospitalização. ${ }^{(19)}$

Estudo com 55 idosos integrantes de equipes domiciliares de cuidados continuados do Sul de Portugal apontou que $40(72,7 \%)$ deles não aderiram à terapêutica medicamentosa. Apesar de não se verificar associação estatística, fatores como baixo nível de rendimento, viver sozinho e ter depressão podem ter influenciado no resultado ${ }^{(19)} \mathrm{e}$, assim, servir de alerta para os profissionais que planejam a alta hospitalar.

O preparo para o autocuidado é primordial para a continuidade do cuidado, pois a educação em saúde necessita ser realizada em todos os âmbitos da atenção à saúde de modo articulado. Na atenção terciária é desenvolvido pelos enfermeiros assistenciais por meio da preparação do paciente e da família durante a internação para o momento da alta do paciente. Programas de preparo para a alta hospitalar, devem ser incentivados, como uma estratégia para a educação e uma contribuição para a integralidade do cuidado. ${ }^{(20)}$

A transferência das informações dos pacientes entre o hospital e os serviços extra-hospitalares evidencia, majoritariamente, a comunicação via e-mail e, ainda, o envolvimento dos pacientes por meio do envio das cartas de alta que estes levam até o serviço de referenciação. Destaca-se que a continuidade no fluxo de comunicação previne transtornos dispensáveis como, por exemplo, a falta de clareza nos comunicados entre profissionais e, ainda, contribui para prevenir a possível admissão em outros serviços. ${ }^{(21)}$

Quando cartas de alta são entregues aos pacientes deve-se considerar que há uma transferência de responsabilidade e espera-se que a pessoa e/ou seu cuidador assuma a transmissão das informações. Esta ação traz riscos de uma transição insatisfatória com a ocorrência de eventos adversos pós-alta e, consequente, reinternação dos pacientes. ${ }^{(22)}$ Acredita-se que o ideal seja a adoção de um meio de comunicação efetivo entre os profissionais, somado a um meio que envolva o paciente e seus familiares, para que esses se sintam parte do processo de continuidade e tenham informações por escrito que poderão ajudá-los nos momentos de incertezas.

Como limitações deste estudo, destaca-se a não inclusão dos documentos que regulamentam a EGA e as atividades dos profissionais das unidades de cuidados continuados, que compõem a RNCCle participam do processo de continuidade do cuidado, o que poderia melhorar o entendimento da gestão de altas nos hospitais estudados.

\section{- CONCLUSÃO}

Os resultados do contexto Português acerca das atividades desenvolvidas pelas enfermeiras da EGA e daquelas que desempenham a função de ligação com a EGA nos impõem algumas reflexões, principalmente porque o SNS português avançou com a criação de uma política de cuidados continuados e a implantação da RNCCI.

Os dados desta pesquisa nos mostram que as atividades de identificação dos pacientes que necessitam de cuidados continuados, o meio de transferir as informações entre os profissionais da equipe assistencial e a EGA e desta à RNCCI não são homogêneas, mesmo que existam orientações nacionais sobre o processo de continuidade de cuidados intra e extra- hospitalar.

Quanto ao planejamento da alta hospitalar, verifica-se que pouco mais da metade é iniciado conforme o preconizado. Nesse sentido, acredita-se que é necessário investigar o contexto de trabalho das enfermeiras assistenciais, ou seja, os recursos humanos disponíveis para tal atividade, a sobrecarga de trabalho dos profissionais e, até mesmo, o enfoque dado pela administração de cada hospital quanto ao planejamento da alta. 
Frente aos elementos que compõem o planejamento da alta hospitalar, verifica-se a preocupação das enfermeiras para além das condições clínicas do paciente e o envolvimento com o cuidador, com os profissionais da USF e RNCCI, aspectos essenciais para pacientes que necessitam de cuidados continuados, pois estes além de tratamento clínico necessitam ser preparados junto ao seu cuidador.

Conhecer a experiência dos profissionais que desempenham a função de ligação na alta hospitalar do paciente, com vistas à continuidade do cuidado,ajudará enfermeiras assistenciais e gerentes a delinear estratégias mais consolidadas, cada um dentro do seu contexto, a fim de enfrentar os desafios da descontinuidade do cuidado, por meio de um apropriado planejamento de alta hospitalar, melhor transferência das informações entre os profissionais, e articulação entre os serviços.

Para pesquisas futuras seria oportuno a inclusão de estudos acerca dos fatores que interferem na identificação e na referenciação dos pacientes do hospital para a RNCCl e demais sistemas de referenciação, sobre o seguimento do paciente após a alta hospitalar, a fim de avaliar possíveis reinternações e, ainda, sobre as atividades das unidades de cuidados continuado da RNCCI.

\section{APOIO FINANCEIRO}

O presente trabalho foi realizado com apoio da Coordenação de Aperfeiçoamento de Pessoal de Nível Superior - Brasil (CAPES) - Código de Financiamento 001.

\section{- REFERÊNCIAS}

1. Ministério da Saúde (Portugal). Decreto-Lei n. 136, de 28 de julho de2015:Procede à primeira alteração ao DecretoLei n. 101/2006, de 6 de junho, que cria a Rede Nacional de Cuidados Continuados Integrados, e à segunda alteração ao Decreto-Lei n. 8/2010, de 28 de janeiro, que cria um conjunto de unidades e equipas de cuidados continuados integrados de saúde mental. Portugal: Diário da República Eletrônico, [Internet] 28 jul2015[acesso em 12 jan 2018 ]. Disponível: https://dre.pt/web/guest/pesquisa/-/search/69879425/details/maximized.

2. Mendes FRP, Gemito MLGP, Caldeira EC, Serra IC, Casas-Novas MV. A continuidade de cuidados de saúde na perspectiva dos utentes. Ciênc. saúde coletiva. [Internet] 2017;22(3) [acesso em 12 jan 2018]. Disponível: http://dx.doi. org/10.1590/1413-81232017223.26292015.

3. Barker I, Steventon A, Deeny SR. Association between continuity of care in general practice and hospital admissions for ambulatory care sensitive conditions: cross sectional study of routinely collected, person level data. BMJ.[Internet] 2017;84(356) [acesso em 12 jan 2018]. Disponível: http://dx.doi.org/10.1136/bmj.j84.

4. Nyweide DJ, Anthony DL, Bynum JP, Strawderman RL, Weeks WB, Casalino LP, et al. Continuity of care and the risk of preventable hospitalization in older adults. JAMA InternMed. [Internet] 2013;173(20) [acesso em 12 jan 2018 ]. Disponível: http://dx.doi.org/10.1001/jamainternmed.2013.10059.

5. Nam YS, Cho KH, Kang HC, Lee KS, Park EC. Greater continuity of care reduces hospital admissions inpatients with hypertension: An analysis of nationwide health insurance data in Korea, 2011-2013. Health Policy.[Internet] 2016;120(6) [acesso em 12 jan 2018]. Disponível: https://doi.org/10.1016/j.healthpol.2016.04.012.

6. Ministério da Saúde (Portugal). Decreto-Lei n. 101, de 06 de junho de 2006 Cria a Rede Nacional de Cuidados Continuados Integrados. Portugal: Diário da República Eletrônico, [Internet]06 jun 2006 [acesso em 12 jan 2018 ]. Disponível: https://dre.pt/pesquisa/-/search/353934/details/maximized.

7. Ministérios das Finanças, da Saúde e da Solidariedade, Emprego e Segurança Social. (Portugal). Portaria n. 174, de 10 de setembro de 2014. Define as condições de instalação e funcionamento a que devem obedecer as unidades de internamento e de ambulatório e as condições de funcionamento das equipas de gestão de altas e as equipas de cuidados continuados integrados da Rede Nacional de Cuidados Continuados Integrados. Portugal: Diário da República Eletrônico, [Internet] 10 set 2014 [acesso em 12 jan 2018]. Disponível: https://dre.pt/web/guest/analisejuridica/-/ aj/56721576/init/normal?p_p_auth=iivSS12h\&_AnaliseJuridica_WAR_drefrontofficeportlet_mode=dt. 
8. Trabalho, Solidariedade e Segurança Social e Saúde (Portugal). Portaria n. 50, de 2 de fevereiro de 2017. Estabelece como prioridade expandir e melhorar a integração da Rede Nacional de Cuidados Continuados Integrados Diário da República Online,02 fev 2017; 608-29.p Portugal.

9. Ribas EN, Bernardino E, Larocca LM, Poli NP, Aued GK, Silva CPC. Enfermeira de ligação: uma estratégia para a contrarreferência. Rev. Bras. Enferm. [Internet] 2018;71(Suppl 1) [acesso em 18 de abr 2018]. Disponível: http://dx.doi. org/10.1590/0034-7167-2017-0490.

10. Nóbrega VM, Reichert APS, Viera CS, Collet N. Longitudinality and continuity of care for children and adolescents with chronic diseases. Esc. Anna Nery. [Internet] 2015;19(4) [acessoem 12 jan 2018]. Disponível: http://dx.doi. org/10.5935/1414-8145.20150088.

11. da Cunha KS, Higashi GDC, Erdmann AL, Kahl C, Koerich C, Meirelles BHS. Myocardial revascularization: factors intervening in the reference and counter-reference in Primary Health Care. Rev. esc. enferm. USP. [Internet] 2016;50(6) [acesso em 12 jan 2018]. Disponível: http://dx.doi.org/10.1590/s0080-623420160000700013.

12. Bernardino E, Segui MLH, Lemos MB, Peres AM. Enfermeira de ligação: uma estratégia de integração em rede. Rev. bras. enferm. [Internet] 2010;63(3) [acesso em12 jan 2018]. Disponível: http://dx.doi.org/10.1590/S003471672010000300018.

13. Health Information and Quality Authority (HIQA). National standard for patient discharge summary information. 2013. [Internet] Ireland: HIQA; 2013 [acesso em 12 jan 2018]. Disponível: https://www.hiqa.ie/system/files/NationalStandard-Patient-Discharge-Summary.pdf.

14. Entidade Reguladora da Saúde (Portugal). Avaliação do acesso dos utentes aos cuidados continuados de saúde. 2013. [Internet] Porto: Entidade Reguladora da Saúde; 2013. [acesso em 12 jan 2018]. Disponível: https://www.ers.pt/ uploads/writer_file/document/824/Cuidados_continuados.pdf.

15. Nogueira JW da S, Rodrigues MCS. Comunicação efetiva no trabalho em equipe em saúde: desafio para a segurança do paciente. Cogitareenferm. [Internet] 2015;20(3) [acesso em 12 jan 2018]. Disponível: http://dx.doi.org/10.5380/ ce.v20i3.40016.

16. Stein-Backes D, Stein-Backes M, Lorenzini-Erdmann A, Büscher A, Salazar-Maya AM. Significado da prática social do enfermeiro com e a partir do Sistema Único de Saúde brasileiro. Aquichan. [Internet] 2014;14(4) [acesso em 12 jan 2018]. Disponível: http://dx.doi.org/10.5294/aqui.2014.14.4.10.

17. Rodrigues RMC, Silva CFR, Loureiro LMJL, Silva SMDT, Crespo SSS, Azeredo ZAS. Os muito idosos: avaliação funcional multidimensional. Rev. Enf. Ref. [Internet] 2015;4(5) [acesso em 12 jan 2018]. Disponível: http://dx.doi. org/10.12707/RIV14040.

18. Ribeiro OMPL, Pinto CAS, Regadas SCRS. A pessoa dependente no autocuidado: implicações para a Enfermagem. Rev. Enf. Ref. [Internet] 2014;4(1) [acesso em 12 jan 2018]. Disponível: http://www.scielo.mec.pt/pdf/ref/vserlVn1/ serIVn1a04.pdf.

19. Monterroso LEP, Joaquim N, Sá LO. Adesão do regime terapêutico medicamentoso dos idosos integrados nas equipas domiciliárias de cuidados continuados. Rev. Enf. Ref. [Internet] 2015;4(5) [acesso em 12 jan 2018]. Disponível: http://dx.doi.org/10.12707/RIV14047.

20. da Costa SRD, de Castro EAB, Acioli S. Self-care ability of hospitalized adults and elderly people: impact on nursing care. REME. [Internet] 2012;17(1) [acesso em 12 jan 2018]. Disponível: http://dx.doi.org/10.5935/1415-2762.20130016.

21. Flesch LD, de Araujo TCCF. Alta hospitalar de pacientes idosos: necessidades e desafios do cuidado contínuo. Estud.psicol. [Internet] 2014;19(3) [acesso em 12 jan 2018]. Disponível: http://dx.doi.org/10.1590/S1413294X2014000300008.

22. Kable A, Chenoweth L, Pond D, Hullick C. Health professional perspectives on systems failures in transitional care for patients with dementia and their carers: a qualitative descriptive study. BMC Health Serv Res. [Internet] 2015;15(567) [acessoem 12 jan 2018]. Disponível: https://doi.org/10.1186/s12913-015-1227-z. 